\title{
Sintomas de depressão, ansiedade, estresse e fatores associados em estudantes de medicina brasileiros: revisão integrativa
}

\author{
Symptoms of depression, anxiety, stress and associated factors in Brazilian medicine students: an \\ integrative review
}

Síntomas de depresión, ansiedad, estrés y factores asociados en estudiantes de medicina brasileña: revisión integrativa

Tâmaro Chagas Mendes

ORCID: https://orcid.org/0000-0003-0645-6706 Universidade Federal dos Vales do Jequitinhonha e Mucuri, Brasil E-mail: tamaro.mendes@ufvjm.edu.br

Ana Catarina Perez Dias

ORCID: https://orcid.org/0000-0002-6690-4139 Universidade Federal dos Vales do Jequitinhonha e Mucuri, Brasil E-mail: anacatarina.dias@ufvjm.edu.br

\begin{abstract}
Resumo
Sintomas de depressão, ansiedade e estresse possuem significativa prevalência em estudantes de medicina brasileiros. Este artigo teve como objetivo identificar as prevalências, os instrumentos de avaliação utilizados e os fatores associados mais citados na literatura científica nacional. Utilizou-se como método uma revisão integrativa da literatura realizada na base de dados Scielo e na Biblioteca Virtual em Saúde (BVS). Encontrou-se nesta revisão grande variação de prevalência de sintomas de depressão, ansiedade e estresse nos estudantes de medicina brasileiros. Depressão foi o transtorno mais avaliado e os sintomas de estresse obtiveram maiores índices de prevalência. Os fatores associados encontrados nesta revisão podem ser classificados em relação às características pessoais dos estudantes; a aspectos familiares e financeiros; à percepção do estudante sobre o curso e ao seu desempenho acadêmico; a fatores que interferem na saúde e na qualidade de vida e a aspectos psicológicos como presença de estresse e histórico de acompanhamento psicológico ou psiquiátrico.
\end{abstract}

Palavras-chave: Estudantes de medicina; Transtornos mentais; Perfil de saúde; Ensino.

\begin{abstract}
Symptoms of depression, anxiety and stress have a significant prevalence in Brazilian medical students. This article aimed to identify the prevalences, the assessment instruments used and the associated factors most cited in the national scientific literature. An integrative literature review carried out in the Scielo database and in the Biblioteca Virtual em Saúde (BVS) was used as a method. This review found a wide variation in the prevalence of symptoms of depression, anxiety and stress in Brazilian medical students. Depression was the most evaluated disorder and the symptoms of stress had higher prevalence rates. The associated factors found in this review can be classified in relation to the students' personal characteristics; family and financial aspects; the student's perception of the course and his academic performance; factors that interfere with health and quality of life; and psychological aspects such as the presence of stress and a history of psychological or psychiatric treatment.
\end{abstract}

Keywords: Medical students; Mental disorders; Health profile; Teaching.

\section{Resumen}

Los síntomas de depresión, ansiedad y estrés tienen una prevalencia significativa en los estudiantes de medicina brasileños. Este artículo tuvo como objetivo identificar las prevalencias, los instrumentos de evaluación utilizados y los factores asociados más citados en la literatura científica nacional. Se utilizó como método una revisión integradora de la literatura realizada en la base de datos Scielo y en la Biblioteca Virtual em Saúde (BVS). Esta revisión encontró una amplia variación en la prevalencia de síntomas de depresión, ansiedad y estrés en estudiantes de medicina brasileños. La depresión fue el trastorno más evaluado y los síntomas del estrés tuvieron mayores tasas de prevalencia. Los factores asociados encontrados en esta revisión se pueden clasificar en relación con las características personales de los estudiantes; aspectos familiares y económicos; la percepción del alumno sobre el curso y su desempeño académico; 
factores que interfieren con la salud y la calidad de vida, y aspectos psicológicos como la presencia de estrés y antecedentes de apoyo psicológico o psiquiátrico.

Palabras clave: Estudiantes de medicina; Trastornos mentales; Perfil de salud; Ensenãnza.

\section{Introdução}

A presença de transtornos mentais influencia os estudos e a qualidade de vida dos estudantes de medicina (Amorin et al., 2018; Santos et al., 2017). Hope et al. (2014) encontraram uma associação entre problemas psicológicos e declínio na performance acadêmica e prejuízos no profissionalismo e na empatia com o paciente.

Sobre a prevalência de transtornos mentais em estudantes de medicina, Pacheco et al. (2017) encontraram prevalências de 30,6\% para depressão; 62,1\% (estado) e 89,6\% (traço) para ansiedade e 49,9\% para estresse. Ainda em relação à depressão, uma meta-análise com 77 países estimulou uma prevalência global de 28\% de prevalência nos estudantes de medicina (Puthran, Zhang, Tam, \& Ho, 2016). Adicionalmente, a suicidalidade (ideação, planejamento, tentativas de suicídio) é uma preocupação crescente associada à depressão em estudantes de medicina (Graner \& Cerqueira, 2019). Sobre essa situação, Akerman (2012) questiona se estresse, ansiedade e depressão nesses estudantes não seria um problema de saúde pública, no sentido que é de interesse de todos enfrentar e solucionar esse problema.

Uma vez que os transtornos mentais apresentam uma etiologia multicausal, é comum que diversos fatores estejam associados ao surgimento dos sintomas. Nos estudantes de medicina, gênero feminino, personalidade mal-adaptativa, dificuldades financeiras e pré-existência de transtornos mentais estão entre os principais fatores associados (Pacheco et al., 2017). De modo geral, contribui também a combinação de características pessoais dos estudantes, tipo de treinamento na faculdade e mecanismos de enfrentamento de estresse (Baldassin, 2012).

Diversas pesquisas identificaram prevalências de sintomas e os fatores associados ao surgimento de depressão, ansiedade e estresse em estudantes de medicina brasileiros. Este artigo teve como objetivo identificar as prevalências, os instrumentos de avaliação utilizados e os fatores associados mais citados na literatura nacional.

\section{Metodologia}

A pesquisa possuiu abordagem quantitativa e foi desenvolvida a partir de uma revisão integrativa conforme as considerações de Souza, Silva e Carvalho (2010). A revisão integrativa da literatura foi realizada na base de dados Scielo e na Biblioteca Virtual em Saúde (BVS), por meio dos descritores "depressão" “ansiedade”, "estresse”, combinados com o termo "estudantes de medicina" com auxílio do operador booleano "and”. Utilizando-se os filtros "Brasil”, "texto completo" e "últimos cinco anos" (2015-2020), obteve-se 101 resultados. Buscou-se apenas por artigos completos e gratuitos. Após a leitura do título e do resumo, eliminou-se artigos presentes nas duas bases de dados, cuja amostra era estrangeira e que objetivaram realizar a validação de instrumentos de avaliação. Foram selecionados 12 artigos ao final desta primeira fase. Utilizando-se os mesmos critérios anteriores, procurou-se por artigos semelhantes nas referências bibliográficas dos artigos anteriormente selecionados. Selecionou-se 3 artigos ao final desta segunda fase. Finalmente, 15 artigos foram selecionados porque atendiam aos objetivos desta pesquisa. A Tabela 1 apresenta o método de seleção dos artigos incluídos nesta revisão. 
Research, Society and Development, v. 10, n. 4, e14910414033, 2021

(CC BY 4.0) | ISSN 2525-3409 | DOI: http://dx.doi.org/10.33448/rsd-v10i4.14033

Tabela 1 - Método de seleção dos artigos incluídos na revisão.

\begin{tabular}{|c|c|c|c|}
\hline Fases da busca & Local de busca dos artigos & Encontrado & Selecionado \\
\hline \multirow{2}{*}{$1^{\text {a } \text { fase }}$} & Scielo & 49 & 10 \\
\cline { 2 - 4 } & BVS & 49 & 02 \\
\hline \multirow{2}{*}{$2^{\text {a fase }}$} & $\begin{array}{c}\text { Referências dos artigos } \\
\text { selecionados (Scielo e BVS) }\end{array}$ & 03 & 03 \\
\hline & Total & 101 & 15 \\
\hline
\end{tabular}

Fonte: Autores (2020).

\section{Resultados}

Em relação às regiões do Brasil onde as pesquisas foram realizadas, 40\% (6) foram na região Sudeste, 26\% (4) na região Nordeste, também 26\% (4) na região Sul e 6\% (1) na região Norte. De modo geral, os artigos tiveram como objetivo principal estimar a prevalência de sintomas de ansiedade, depressão e estresse. A amostra das pesquisas foi composta em média por 327 estudantes de graduação em medicina.

As prevalências encontradas nas pesquisas variaram entre 5,6\% a 45,7\% para depressão (Oliveira et al., 2016; Vasconcelos et al., 2015); 13,4\% a 41,4\% para ansiedade (Ribeiro et al., 2020; Taborda, 2015) e 40,95\% a 78,98\% para estresse (Bassols et al., 2015a; Estrela, Rezende, Guedes, Pereira, \& Sousa, 2018).

Encontrou-se um total de 07 instrumentos utilizados pelas pesquisas selecionadas nesta revisão. Os instrumentos mais utilizados para avaliar depressão, ansiedade e estresse foram, respectivamente, o Inventário Beck de Depressão (Beck Depression Inventory - BDI), utilizado em 08 pesquisas; o Inventário de Ansiedade de Beck (Beck Anxiety Inventory - BAI), utilizado em 05 pesquisas; a Escala Hospitalar de Ansiedade e Depressão (Hospital Anxiety and Depression Scale - HAD), utilizada em 02 pesquisas; o Questionário de Saúde Geral de Goldberg (QSG) (General Health Questionnaire - GHQ-12), utilizado também em 02 pesquisas; a Escala de Depressão, Ansiedade e Estresse (Depression, Anxiety and Stress - DASS-21), utilizada em 01 pesquisa; o Inventário de Ansiedade Traço-Estado (IDATE) (Stait-Trait Anxiety Inventory - STAI); também utilizado em 01 pesquisa; o Inventário de Sintomas de Stress para Adultos de Lipp (ISSL), utilizado em 5 pesquisas, e a Escala de Estresse Percebido (Perceived Stress Scale - PSS) utilizada em 01 pesquisa.

A Tabela 2 apresenta os artigos utilizados nesta revisão, os instrumentos e as prevalências encontradas em cada pesquisa.

Tabela 2 - Artigos incluídos na revisão.

\begin{tabular}{|c|c|c|c|c|c|}
\hline Autor(es) & Ano & Local & Objetivo & Instrumentos & Prevalência encontrada \\
\hline Costa et al. & 2020 & $\mathrm{RN}$ & $\begin{array}{l}\text { Estimar a prevalência de } \\
\text { sintomas de estresse, } \\
\text { depressão e ansiedade } \\
\text { dos estudantes de } \\
\text { Medicina da } \\
\text { Universidade Federal do } \\
\text { Rio Grande do Norte } \\
\text { (UFRN), associando-os } \\
\text { com outros fatores. }\end{array}$ & $\begin{array}{l}\text { Inventário de } \\
\text { Depressão de Beck } \\
\text { (IDB); Inventário } \\
\text { de Ansiedade de } \\
\text { Beck (BAI) e } \\
\text { Inventário de } \\
\text { Sintomas de Stress } \\
\text { para Adultos de } \\
\text { Lipp (ISSL) }\end{array}$ & $\begin{array}{l}\text { Neste estudo, verificou-se a } \\
\text { presença de estresse em } 66,3 \% \\
\text { dos estudantes, sintomas ansiosos } \\
\text { em } 33,6 \% \text { e sintomas depressivos } \\
\text { em } 28 \% \text {. }\end{array}$ \\
\hline
\end{tabular}




\begin{tabular}{|c|c|c|c|c|c|}
\hline Ribeiro et al. & 2020 & RJ & $\begin{array}{l}\text { (a) estimar a prevalência } \\
\text { de escores indicativos de } \\
\text { ansiedade, depressão e } \\
\text { para ansiedade e } \\
\text { depressão simultâneas e } \\
\text { (b) analisar fatores } \\
\text { associados a essas } \\
\text { condições em estudantes } \\
\text { de medicina de uma } \\
\text { universidade federal no } \\
\text { Brasil. }\end{array}$ & $\begin{array}{l}\text { Hospital Anxiety } \\
\text { and Depression } \\
\text { Scale (HADS) }\end{array}$ & $\begin{array}{l}\text { A ansiedade foi a condição mais } \\
\text { comum }(41,4 \%) \text {, seguida por } \\
\text { depressão }(8,2 \%) \text {, e ansiedade e } \\
\text { depressão simultaneamente } \\
(7,0 \%) .\end{array}$ \\
\hline Kam et al. & 2020 & SP & $\begin{array}{l}\text { Avaliar a ocorrência do } \\
\text { estresse durante a } \\
\text { graduação médica em } \\
\text { acadêmicos de uma } \\
\text { universidade privada da } \\
\text { região do Alto Tietê, no } \\
\text { Estado de São Paulo }\end{array}$ & $\begin{array}{l}\text { Inventário de } \\
\text { Sintomas de } \\
\text { Estresse para } \\
\text { Adultos de Lipp } \\
\text { (ISSL) e a Escala } \\
\text { de Estresse } \\
\text { Percebido (PSS) }\end{array}$ & $\begin{array}{l}\text { O ISSL demonstrou a ocorrência } \\
\text { de estresse em } 65 \% \text { dos } \\
\text { estudantes. }\end{array}$ \\
\hline $\begin{array}{l}\text { Medeiros et } \\
\text { al. }\end{array}$ & 2018 & $\mathrm{MG}$ & $\begin{array}{l}\text { Avaliar globalmente a } \\
\text { saúde mental dos } \\
\text { acadêmicos ingressantes } \\
\text { no curso médico, com } \\
\text { ênfase em qualidade de } \\
\text { vida, Transtornos } \\
\text { Mentais Comuns, } \\
\text { sintomas depressivos, } \\
\text { nível de sonolência } \\
\text { diurna e Burnout, } \\
\text { segundo o sexo }\end{array}$ & $\begin{array}{l}\text { Escala de } \\
\text { Sonolência de } \\
\text { Epworth15; } \\
\text { Inventário de } \\
\text { Depressão de } \\
\text { Beck; Short-Form } \\
\text { Health Survey (SF- } \\
\text { 12); Questionário } \\
\text { de Saúde Geral } \\
\text { (QSG-12); } \\
\text { Maslach Burnout } \\
\text { Inventory-Student } \\
\text { Survey (MBI-SS) }\end{array}$ & $\begin{array}{l}\text { O estresse esteve presente em } \\
45,5 \% \text { dos estudantes. A } \\
\text { prevalência de sintomas } \\
\text { depressivos em grau variado } \\
\text { também foi significativa, afetando } \\
43,6 \% \text {. }\end{array}$ \\
\hline Estrela et al. & 2018 & PB & $\begin{array}{l}\text { Analisar as fases do } \\
\text { estresse e seus correlatos } \\
\text { com características de } \\
\text { saúde e demográficas de } \\
\text { estudantes de medicina } \\
\text { de uma faculdade do } \\
\text { interior da Paraíba. }\end{array}$ & $\begin{array}{l}\text { Inventário de } \\
\text { Sintomas de Stress } \\
\text { para Adultos de } \\
\text { Lipp (ISSL) }\end{array}$ & $\begin{array}{l}21,02 \% \text { dos acadêmicos não } \\
\text { apresentavam estresse e 78,98\% } \\
\text { apresentavam. }\end{array}$ \\
\hline Rollemberg & 2018 & $\mathrm{NE}$ & $\begin{array}{l}\text { Determinar a presença de } \\
\text { sintomas } \\
\text { de ansiedade e depressão } \\
\text { nos estudantes de } \\
\text { Medicina da UFS- } \\
\text { Campus Lagarto e } \\
\text { possíveis fatores } \\
\text { associados. }\end{array}$ & $\begin{array}{l}\text { Inventário de } \\
\text { Depressão de Beck } \\
\text { (IDB); Inventário } \\
\text { de Ansiedade de } \\
\text { Beck (BAI) }\end{array}$ & $\begin{array}{l}\text { Os resultados principais } \\
\text { evidenciaram que } 25,87 \% \text { dos } \\
\text { estudantes apresentaram algum } \\
\text { grau de ansiedade de leve a grave, } \\
\text { e } 24,47 \% \text { de depressão de leve a } \\
\text { grave. }\end{array}$ \\
\hline $\begin{array}{l}\text { Moutinho et } \\
\text { al. }\end{array}$ & 2017 & MG & $\begin{array}{l}\text { Comparar a prevalência } \\
\text { de ansiedade, depressão e } \\
\text { estresse em estudantes de } \\
\text { medicina de todos os } \\
\text { semestres de uma } \\
\text { faculdade de medicina } \\
\text { brasileira e avaliar seus } \\
\text { respectivos fatores } \\
\text { associados }\end{array}$ & $\begin{array}{l}\text { Depression, } \\
\text { Anxiety, and Stress } \\
\text { Scale (DASS-21) }\end{array}$ & $\begin{array}{l}34,6 \% \text { relataram sintomatologia } \\
\text { depressiva, } 37,2 \% \text { apresentaram } \\
\text { sintomas de ansiedade e } 47,1 \% \\
\text { sintomas de estresse. }\end{array}$ \\
\hline
\end{tabular}




\begin{tabular}{|c|c|c|c|c|c|}
\hline $\begin{array}{l}\text { Cybulski e } \\
\text { Mansani }\end{array}$ & 2017 & PR & $\begin{array}{l}\text { Determinar a prevalência } \\
\text { de sintomas depressivos e } \\
\text { de seus fatores de risco, } \\
\text { assim como do uso de } \\
\text { antidepressivos na } \\
\text { amostra analisada. }\end{array}$ & $\begin{array}{l}\text { Inventário de } \\
\text { Depressão de Beck } \\
\text { (IDB); }\end{array}$ & $\begin{array}{l}\text { Prevalência total de depressão: } \\
44,22 \% \text {. }\end{array}$ \\
\hline Lima et al. & 2016 & PR & $\begin{array}{l}\text { Verificar a prevalência do } \\
\text { estresse entre estudantes } \\
\text { de Medicina, a relação } \\
\text { entre morar ou não com a } \\
\text { família e sua repercussão } \\
\text { sobre o rendimento } \\
\text { acadêmico }\end{array}$ & $\begin{array}{l}\text { Inventário de } \\
\text { Sintomas de } \\
\text { Estresse para } \\
\text { Adultos de Lipp }\end{array}$ & $\begin{array}{l}\text { Verificou-se a presença de } \\
\text { estresse em } 60,09 \% \text { da amostra. }\end{array}$ \\
\hline Oliveira et al & 2016 & $\mathrm{AP}$ & $\begin{array}{l}\text { Conhecer a prevalência e } \\
\text { os fatores associados aos } \\
\text { sintomas depressivos em } \\
\text { estudantes de medicina } \\
\text { da Universidade Federal } \\
\text { do Amapá (UNIFAP) }\end{array}$ & $\begin{array}{l}\text { Inventário de } \\
\text { Depressão de Beck } \\
\text { (IDB) e DREEM } \\
\text { (Dundee Ready } \\
\text { Education } \\
\text { Environment } \\
\text { Measurement) }\end{array}$ & $\begin{array}{l}\text { 45,7\% apresentaram algum grau } \\
\text { de depressão. }\end{array}$ \\
\hline $\begin{array}{l}\text { Serra, Dinato } \\
\text { e Caseiro }\end{array}$ & 2015 & SP & $\begin{array}{l}\text { Verificar a prevalência de } \\
\text { sintomas depressivos e } \\
\text { ansiosos em estudantes } \\
\text { de medicina, } \\
\text { considerando dados da } \\
\text { literatura que indicam } \\
\text { maior vulnerabilidade a } \\
\text { transtornos emocionais } \\
\text { nessa população. }\end{array}$ & $\begin{array}{l}\text { Inventário de } \\
\text { Depressão de Beck } \\
\text { (IDB); Inventário } \\
\text { de Ansiedade de } \\
\text { Beck (BAI) }\end{array}$ & $\begin{array}{l}30 \% \text { apresentaram sintomas } \\
\text { depressivos e } 21 \% \text { sintomas de } \\
\text { ansiedade. }\end{array}$ \\
\hline $\begin{array}{l}\text { Tabalipa et } \\
\text { al. }\end{array}$ & 2015 & $\mathrm{SC}$ & $\begin{array}{l}\text { Estimar a prevalência e } \\
\text { os fatores associados de } \\
\text { ansiedade e depressão } \\
\text { entre estudantes de } \\
\text { medicina. }\end{array}$ & $\begin{array}{l}\text { Inventário de } \\
\text { Depressão de Beck } \\
\text { (IDB); Inventário } \\
\text { de Ansiedade de } \\
\text { Beck (BAI) }\end{array}$ & $\begin{array}{l}\text { A prevalência de ansiedade foi de } \\
35,5 \% \text { e depressão foi de } 32,8 \% \text {. }\end{array}$ \\
\hline $\begin{array}{l}\text { Vasconcelos } \\
\text { et al. }\end{array}$ & 2015 & $\mathrm{PE}$ & $\begin{array}{l}\text { Determinar a prevalência } \\
\text { de sintomas de ansiedade } \\
\text { e depressão em } \\
\text { estudantes de Medicina e } \\
\text { avaliar fatores } \\
\text { associados. } \\
\end{array}$ & $\begin{array}{l}\text { Escala Hospitalar } \\
\text { de Ansiedade e } \\
\text { Depressão (Ehad). }\end{array}$ & $\begin{array}{l}\text { Ansiedade: } 19,7 \% \text { manifestando } \\
\text { sintomas sugestivos do } \\
\text { transtorno. Depressão: } 5,6 \% \\
\text { manifestando sintomas sugestivos } \\
\text { do transtorno. }\end{array}$ \\
\hline Taborda & 2015 & SP & $\begin{array}{l}\text { Estimar a prevalência de } \\
\text { depressão e ansiedade } \\
\text { entre os alunos do } 1^{\circ} \text { ao } \\
6^{\circ} \text { ano }\end{array}$ & $\begin{array}{l}\text { Teste de } \\
\text { apercepção } \\
\text { temática; } \\
\text { Inventário de } \\
\text { Depressão de Beck } \\
\text { (IDB); Inventário } \\
\text { de Ansiedade de } \\
\text { Beck (BAI) }\end{array}$ & $\begin{array}{l}\text { Em relação à ansiedade, } 13,4 \% \\
\text { apresentaram sintomas em nível } \\
\text { leve e } 5,5 \% \text { em nível Moderado. } \\
\text { Em relação à depressão, } 16,0 \% \\
\text { indicaram nível leve e } 4,1 \% \text { nível } \\
\text { moderado. }\end{array}$ \\
\hline Bassols et al & 2015 & $\mathrm{RS}$ & $\begin{array}{l}\text { Avaliar a prevalência de } \\
\text { estresse e coping em } \\
\text { estudantes de uma escola } \\
\text { pública de medicina do } \\
\text { Brasil, comparando os } \\
\text { grupos do primeiro e do } \\
\text { sexto ano de formação. }\end{array}$ & $\begin{array}{l}\text { Inventário de } \\
\text { Sintomas de } \\
\text { Estresse para } \\
\text { Adultos de Lipp }\end{array}$ & $\begin{array}{l}\text { Na amostra, 40,95\% dos sujeitos } \\
\text { (95 alunos) apresentaram } \\
\text { sintomas de estresse. }\end{array}$ \\
\hline
\end{tabular}

Fonte: Autores (2020). 
Research, Society and Development, v. 10, n. 4, e14910414033, 2021

(CC BY 4.0) | ISSN 2525-3409 | DOI: http://dx.doi.org/10.33448/rsd-v10i4.14033

\section{Fatores associados à depressão}

Foi relevante à associação entre depressão e sexo feminino (Medeiros, Camargo, Barbosa, \& Caldeira, 2018; Rollemberg, 2018; Serra, Dinato, \& Caseiro, 2015; Tabalipa et al., 2015), ser jovem (18 a 25 anos), solteiro, estar no segundo ano do curso (Rollemberg, 2018); ter estudado o ensino médio em escola pública, sentir-se cobrado pelo curso, horas reduzidas de sono (Costa et al., 2020); sentir-se sozinho e moralmente prejudicado na faculdade (Ribeiro et al., 2020); baixa frequência de atividades de lazer, baixa satisfação com o desempenho acadêmico, falta de apoio emocional no ambiente acadêmico (Cybulski \& Mansani, 2017); preocupação com o futuro, sentir-se pressionado pelos pais (Tabalipa et al., 2015); uso de drogas ilícitas, ser originário de outra cidade e esteja afastados do âmbito familiar (Vasconcelos et al., 2015); estar no ciclo clínico (Taborda, 2015); desejar mudar de curso, possuir dificuldade de relacionamento social, percepção negativa do ambiente de ensino (Oliveira et al., 2016); apresentar estresse e sintomas de exaustão (Costa et al., 2020; Cybulski \& Mansani, 2017) e ter realizado acompanhamento psicológico ou psiquiátrico (Ribeiro et al., 2020; Vasconcelos et al., 2015).

\section{Fatores associados à ansiedade}

Quanto à ansiedade, foi relevante à associação com o sexo feminino (Costa et al., 2020; Ribeiro et al., 2020; Rollemberg, 2018; Tabalipa et al., 2015; Taborda, 2015); ser jovem (18 a 25 anos), ser solteiro e estar no quinto ano (Rollemberg, 2018); sentir-se sozinho e moralmente prejudicado (Ribeiro et al., 2020); dormir menos horas por dia (Costa et al., 2020); estudantes nos períodos iniciais ou nos primeiros ciclos do curso (Costa et al., 2020; Moutinho et al., 2017; Rollemberg, 2018; Serra et al., 2015; Taborda, 2015); sentir-se pressionado pelos pais (Tabalipa et al., 2015); uso de drogas psicoativas (Vasconcelos et al., 2015) e ter realizado acompanhamento psicológico (Costa et al., 2020; Ribeiro et al., 2020; Vasconcelos et al., 2015).

\section{Fatores associados ao estresse}

O estresse esteve associado ao sexo feminino (Bassols et al., 2015b; Kam et al., 2019); estudantes mais jovens (17 a 24 anos) e com menor satisfação com o curso (Bassols et al., 2015b; Estrela et al., 2018); estar nos períodos iniciais (Bassols et al., 2015b; Estrela et al., 2018; Kam et al., 2019) transição entre os estilos de vida, grande volume de informações para compreender, conciliar o limitado tempo para estudar com a realização das atividades de lazer e domésticas, competição entre os estudantes, questões íntimas, aspectos familiares, vestibular competitivo, estranhamento da metodologia de ensino da faculdade, ciclo básico sem contato com atividades mais próximas da prática médica (Lima, Soares, Prado, \& Albuquerque, 2016); estudantes sem bolsa de estudo, não procurar atendimento psicológico (Estrela et al., 2018); aspectos financeiros e usar estratégia de coping evitativo (Bassols et al., 2015b; Lima et al., 2016).

\section{Discussão}

As pesquisas encontradas nesta revisão foram de caráter transversal, quantitativa e objetivaram identificar a prevalência de sintomas de depressão, ansiedade e estresse e os fatores associados. No entanto, a pesquisa de Lima et al (2016) utilizou grupo focal como uma estratégia qualitativa.

Os instrumentos utilizados foram questionários validados e adaptados para o português brasileiro e que possuíam boas características psicométricas para a população estudada. Os instrumentos mais utilizados pelos pesquisadores brasileiros foram os mesmos mais utilizados por pesquisadores internacionais pesquisando estudantes de medicina (Hope \& Henderson, 2014).

Encontrou-se nesta revisão diversos valores de prevalência. Essa variação na prevalência pode ocorrer devido às características sociodemográficas e acadêmicas da população estudada, características das instituições de ensino e escolhas 
Research, Society and Development, v. 10, n. 4, e14910414033, 2021

(CC BY 4.0) | ISSN 2525-3409 | DOI: http://dx.doi.org/10.33448/rsd-v10i4.14033

metodológicas das pesquisas (critérios de inclusão e exclusão, instrumentos de pesquisa, análise dos dados etc.). Os cursos da saúde, principalmente medicina, estão entre os mais pesquisados em relação à saúde mental dos estudantes universitários (Leão, Gomes, Ferreira, \& Cavalcanti, 2018). Existe preocupação especialmente em relação aos estudantes de medicina e em médicos, pois possuem taxas de suicídio maiores do que as da população geral e de outros grupos acadêmicos (Santa, Cantilino, Santa, \& Cantilino, 2016). Em relação aos estudantes de medicina, são fatores de risco associados à tentativa de suicídio ser do sexo feminino; ser homossexual; menor renda; bullying na Universidade; história de trauma na infância ou na idade adulta; história familiar positiva para suicídio; ter tido ideação suicida no último mês; uso diário de tabaco e estar sob risco severo de abuso de álcool (Marcon et al., 2020).

Os fatores associados à depressão, ansiedade e estresse encontrados nesta revisão podem ser classificados em relação às características pessoais dos estudantes; a aspectos familiares e financeiros; à percepção do estudante sobre o curso e seu desempenho acadêmico; a fatores que interferem na saúde e na qualidade de vida do estudante (uso de drogas, horas de sono e de atividades de lazer, p. ex) e a aspectos psicológicos como presença de estresse e histórico de acompanhamento psicológico ou psiquiátrico.

Em relação às características pessoais, relevou-se como fatores associados, ser do sexo feminino e jovem. Ressalta-se, entretanto, que as mulheres compuseram a maioria dos estudantes da amostra das pesquisas selecionadas nesta revisão. Apesar disso, pesquisas anteriores também já demonstraram a alta prevalência de transtornos mentais entre as estudantes do sexo feminino quando comparadas aos estudantes do sexo masculino (Medeiros et al., 2018; Pacheco et al., 2017). Na população em geral, os transtornos mentais também tendem a ser mais prevalentes nas as mulheres, possivelmente devido a fatores hormonais, psicológicos, características dos instrumentos de avaliação e métodos de pesquisa (Santos; Siqueira, 2010).

Quanto aos fatores relacionados à idade, encontrou-se associação em ser jovem entre 17 e 25 anos. A adolescência e o início da vida adulta são fases da vida em que grandes transformações, tanto fisiológicas quanto sociais, começam a se manifestar, o que pode aumentar as chances do desenvolvimento de alterações psicopatológicas e ao surgimento de sintomas de depressão e ansiedade (Grolli, Wagner, \& Dalbosco, 2017). Adicionalmente, o estudante jovem pode vivenciar na universidade o que o sociólogo francês Allain Coullon chamou de o tempo de estranheza, no qual o estudante encontra-se distante de sua família e de sua antiga rotina de estudos e agora necessita adaptar-se à nova realidade com cidade, instituição, métodos de ensino e professores diferentes (Coulon, 2017). É importante ressaltar que assim como ocorre em relação ao sexo, os estudantes jovens também eram maioria nas pesquisas. Isso pode confirmar a tendência de feminização e juvenização da medicina (Scheffer, Cassenote, Guilloux, Miotto, \& Mainardi, 2018).

Quanto aos aspectos familiares, sentir-se pressionado pelos pais foi associado apenas ao surgimento de depressão e ansiedade (Tabalipa et al., 2015), enquanto que estar distante da família esteve associado ao estresse (Lima et al., 2016). De fato, os pais possuem um importante papel na vida do estudante, sendo fonte de apoio financeiro e emocional. A pressão percebida pelo estudante pode ter origem tanto nas cobranças dos pais como em seu próprio desejo de atender as expectativas dos seus genitores.

O aspecto financeiro foi associado ao surgimento de estresse nos estudantes com baixa renda e que não possuíam bolsas de estudo (Bassols et al., 2015b; Estrela et al., 2018; Lima et al., 2016). Isso pode demonstrar que as dificuldades financeiras influenciam não somente o sustendo do estudante, mas também a sua saúde mental. Assim, mesmo o curso de medicina ainda sendo caracterizado por ter estudantes de alta renda e classe média (Veras, Fernandez, Feitosa, \& Fernandes, 2020), é preciso pensar em políticas institucionais para apoiar os estudantes de baixa renda.

Fatores associados que estão relacionados ao curso dizem respeito à percepção do estudante sobre o seu desempenho, satisfação e fases do curso (ciclo básico ou ciclo clínico). Sobre essas fases, possivelmente, há fatores diferentes em cada uma, 
Research, Society and Development, v. 10, n. 4, e14910414033, 2021

(CC BY 4.0) | ISSN 2525-3409 | DOI: http://dx.doi.org/10.33448/rsd-v10i4.14033

como, no início do curso: adaptação à faculdade e às novas metodologias de ensino; no ciclo clínico: exposição ao contato com o paciente, com o sofrimento humano e com a morte; e ao final do curso: preocupação com o futuro profissional, com a inserção no mercado de trabalho e em preparar-se para as provas de residência (André et al., 2019).

Aspectos relacionados à qualidade de vida como frequência de atividades de lazer, horas de sono e uso de drogas ilícitas e psicoativas também estão associados ao surgimento de sintomas de depressão, ansiedade e estresse. Amorim et al (2018) classificaram os estudantes de medicina de sua pesquisa com qualidade de vida regular, sendo que $72 \%$ possuíam má qualidade do sono e $17 \%$ possível distúrbio do sono. A pressão por bons resultados e a quantidade de matérias para estudar pode fazer com que os estudantes diminuam sua quantidade e qualidade do sono. Em relação ao uso de álcool e drogas, há evidências de relação entre o consumo de álcool, cuja ingestão aumenta no decorrer dos períodos do curso, e de outras drogas entorpecentes, como o tabaco e a maconha, droga ilícita mais utilizada (Machado, Moura, \& Almeida, 2015).

Outro fator associado relevante foi ter realizado acompanhamento psicológico ou psiquiátrico. Isso pode demonstrar a existência de um sofrimento psíquico prévio, uma vez que estudantes de medicina com transtornos mentais tendem a ter um histórico com problemas mentais antes do início do curso (Pacheco et al., 2017). Para ofertar ajuda para seus estudantes, algumas faculdades de medicina possuem serviços de apoio psicológico (Baldassin, 2012), todavia, acredita-se que alguns estudantes ainda possam ter preconceito ou vergonha em relação às doenças psiquiátricas e às dificuldades psicológicas, o que consequentemente os impediria de utilizar esses serviços (Millan \& Arruda, 2008).

As pesquisas sobre a prevalência de sintomas de depressão, ansiedade e estresse e os fatores associados trazem uma importante contribuição para a compreensão do sofrimento psíquico dos estudantes de medicina. Essas contribuições ocorrem mesmo a despeito de possíveis limitações, como uso de questionários autorreferidos, no qual precisa-se contar com a percepção e a colaboração do participante, e o viés de prevalência-incidência (Viés de Neyman), posto a possível existência de estudantes em tratamento psicológico ou e em estados graves e que abandonaram o curso e assim não entram nos dados coletados (Pacheco et al., 2017).

\section{Considerações Finais}

Encontrou-se nesta revisão grande variação de prevalência de sintomas de depressão, ansiedade e estresse nos estudantes de medicina brasileiros. Depressão foi o transtorno mais avaliado e os sintomas de estresse obtiveram maiores índices de prevalência.

Os fatores associados descritos nesta revisão não podem ser compreendidos como causas únicas ou diretas dos sintomas relatados pelos estudantes. Não obstante, ter ciência sobre esses fatores pode auxiliar os serviços de apoio psicológico das instituições a proporem ações de prevenção e promoção de saúde para os estudantes de medicina. Recomenda-se especial atenção aos fatores relacionados às características pessoais dos estudantes (estudantes jovens e do sexo feminino); aos aspectos familiares (estudantes distantes de casa ou que se sintam pressionados pelos pais); aos aspectos financeiros (estudantes de baixa renda e sem bolsa de estudos); à percepção do estudante sobre o curso e seu desempenho acadêmico; à qualidade de vida (uso de álcool e drogas, horas de sono e frequência de atividades de lazer) e aos aspectos psicológicos como presença de estresse e histórico de acompanhamento psicológico ou psiquiátrico.

Esta revisão identificou as prevalências de depressão, ansiedade e estresse nos estudantes de medicina brasileiros e apresentou uma classificação e discussão sobre os fatores associados mais citados na literatura nacional. Algumas limitações desta revisão concernem-se à utilização de apenas duas bases de dados nacionais e período de publicação dos artigos. Recomenda-se, portanto, cautela na generalização dos resultados apresentados. 
Research, Society and Development, v. 10, n. 4, e14910414033, 2021

(CC BY 4.0) | ISSN 2525-3409 | DOI: http://dx.doi.org/10.33448/rsd-v10i4.14033

Para trabalhos futuros, sugere-se a utilização combinada de métodos quantitativos e qualitativos para que se possa identificar prevalências, fatores associados e possíveis explicações sobre o sofrimento psíquico dos estudantes durante o curso de medicina.

\section{Referências}

Akerman, M. (2012). Estresse, ansiedade e depressão em estudantes de Medicina. In Baldassin, S (Coord). Atendimento psicológico aos estudantes de medicina: técnica e ética. São Paulo: Edipro.

Amorim, B. B., Moraes, L., Sá, I. C. G., \& Silva, Barbara Borges Gomes Camara Filho, J. W. S. (2018). Saúde mental do estudante de Medicina: psicopatologia, estresse, sono e qualidade de vida. Revista Psicologia, Diversidade e Saúde, 7(2), 245.

André, J. C., Melo, J. C. R. de, Lima, A. R. de A., Brienze, S. L. A., Werneck, A. L., \& Fucuta, P. da S. (2019). Cursos Preparatórios para os Exames de Residência e a Evasão dos Cenários de Prática: Cadê o Interno que Estava Aqui? Revista Brasileira de Educação Médica, 43(1), $105-114$.

Baldassin, S. (2012). Atendimento psicológico aos estudantes de medicina: técnica e ética. Edipro.

Bassols, A. M. S., Carneiro, B. B., Guimarães, G. C., Okabayashi, L. M. S., Carvalho, F. G., da Silva, A. B., \& Eizirik, C. L. (2015a). Stress and coping in a sample of medical students in Brazil. Revista de Psiquiatria Clinica, 42(1), 1-5.

Bassols, A. M. S., Carneiro, B. B., Guimarães, G. C., Okabayashi, L. M. S., Carvalho, F. G., da Silva, A. B., \& Eizirik, C. L. (2015b). Stress and coping in a sample of medical students in Brazil. Revista de Psiquiatria Clinica, 42(1), 1-5.

Costa, D. S. da, Medeiros, N. de S. B., Cordeiro, R. A., Frutuoso, E. de S., Lopes, J. M., \& Moreira, S. da N. T. (2020). Sintomas de Depressão, Ansiedade e Estresse em Estudantes de Medicina e Estratégias Institucionais de Enfrentamento. Revista Brasileira de Educação Médica, 44(1).

Coulon, A. (2017). O ofício de estudante: a entrada na vida universitária. Educação e Pesquisa, 43(4), 1239-1250.

Cybulski, C. A., \& Mansani, F. P. (2017). Análise da Depressão, dos Fatores de Risco para Sintomas Depressivos e do Uso de Antidepressivos entre Acadêmicos do Curso de Medicina da Universidade Estadual de Ponta Grossa. Revista Brasileira de Educação Médica, 41(1), 92-101.

Estrela, Y. da C. A., Rezende, A. C. C., Guedes, A. F., Pereira, C. de O., \& Sousa, M. N. A. de. (2018). Estresse e correlatos com características de saúde e sociodemográficas de estudantes de medicina TT - Estrés y características socio-demográficas y de salud en estudiantes de medicina. CES Medicina, 32(3), 215225.

Graner, K. M., \& Cerqueira, A. T. D. A. R. (2019). Revisão integrativa: sofrimento psíquico em estudantes universitários e fatores associados Integrative. Ciencia e Saude Coletiva, 24(4), 1327-1346.

Grolli, V., Wagner, M. F., \& Dalbosco, S. N. P. (2017). Sintomas Depressivos e de Ansiedade em Adolescentes do Ensino Médio. Revista de Psicologia da IMED, 9(1), 87 .

Hope, V., \& Henderson, M. (2014). Medical student depression, anxiety and distress outside North America: a systematic review. Medical education, 48(10), 963-979.

Kam, S. X. L., Toledo, A. L. S. de, Pacheco, C. C., Souza, G. F. B. de, Santana, V. L. M., Bonfá-Araujo, B., \& Custódio, C. R. da S. N. (2019). Estresse em Estudantes ao longo da Graduação Médica. Revista Brasileira de Educação Médica, 43(1 suppl 1), $246-253$.

Leão, A. M., Gomes, I. P., Ferreira, M. J. M., \& Cavalcanti, L. P. de G. (2018). Prevalência e Fatores Associados à Depressão e Ansiedade entre Estudantes Universitários da Área da Saúde de um Grande Centro Urbano do Nordeste do Brasil. Revista Brasileira de Educação Médica, 42(4), 55-65.

Lima, R. L. de, Soares, M. E. C., Prado, S. N. do, \& Albuquerque, G. S. C. de. (2016). Estresse do Estudante de Medicina e Rendimento Acadêmico. Revista Brasileira de Educação Médica, 40(4), 678-684.

Machado, C. de S., Moura, T. M. de, \& Almeida, R. J. de. (2015). Estudantes de Medicina e as Drogas: Evidências de um Grave Problema. Revista Brasileira de Educação Médica, 39(1), 159-167.

Marcon, G., Massaro Carneiro Monteiro, G., Ballester, P., Cassidy, R. M., Zimerman, A., Brunoni, A. R., Passos, I. C. (2020). Who attempts suicide among medical students? Acta Psychiatrica Scandinavica, 141(3), 254-264. h

Medeiros, M. R. B., Camargo, J. F., Barbosa, L. A. R., \& Caldeira, A. P. (2018). Saúde Mental de Ingressantes no Curso Médico: uma Abordagem segundo o Sexo. Revista Brasileira de Educação Médica, 42(3), 214-221.

Millan, L. R., \& Arruda, P. C. V. de. (2008). Assistência psicológica ao estudante de medicina: 21 Anos de experiência. Revista da Associacao Medica Brasileira, $54(1), 90-94$.

Moutinho, I. L. D., Maddalena, N. de C. P., Roland, R. K., Lucchetti, A. L. G., TibiriçÁ, S. H. C., Ezequiel, O. D. S., \& Lucchetti, G. (2017). Depression, stress and anxiety in medical students: A cross-sectional comparison between students from different semesters. Revista da Associacao Medica Brasileira, 63(1), 2128 .

Oliveira, G. S. de, Rocha, C. A., Santos, B. É. F. dos, Sena, I. S., Fávaro, L., \& Guerreiro, M. C. (2016). Prevalência e fatores associados à depressão em estudantes de medicina da Universidade Federal do Amapá. In Revista de Medicina e Saúde de Brasília (Vol. 5). 
Research, Society and Development, v. 10, n. 4, e14910414033, 2021

(CC BY 4.0) | ISSN 2525-3409 | DOI: http://dx.doi.org/10.33448/rsd-v10i4.14033

Pacheco, J. P. G., Giacomin, H. T., Tam, W. W., Ribeiro, T. B., Arab, C., Bezerra, I. M., \& Pinasco, G. C. (2017). Mental health problems among medical students in Brazil: A systematic review and meta-analysis. Revista Brasileira de Psiquiatria, 39(4), 369-378.

Puthran, R., Zhang, M. W. B., Tam, W. W., \& Ho, R. C. (2016). Prevalence of depression amongst medical students: A meta-analysis. Medical Education, 50(4), $456-468$.

Ribeiro, C. F., Lemos, C. M. C., Alt, N. N., Marins, R. L. T., Corbiceiro, W. C. H., \& Nascimento, M. I. do. (2020). Prevalence of and Factors Associated with Depression and Anxiety in Brazilian Medical Students. Revista Brasileira de Educação Médica, 44(1).

Rollemberg, G. de S. M. (2018). Avaliação da presença de sintomas de ansiedade e depressão em estudantes de medicina da Universidade Federal de Sergipe - Campus Lagarto. Universidade Federal de Sergipe.

Santa, N. Della, Cantilino, A., Santa, N. Della, \& Cantilino, A. (2016). Suicídio entre Médicos e Estudantes de Medicina: Revisão de Literatura. Revista Brasileira de Educação Médica, 40(4), 772-780.

Santos, É. G. dos, \& Siqueira, M. M. de. (2010). Prevalência dos transtornos mentais na população adulta brasileira: Uma revisão sistemática de 1997 a 2009. Jornal Brasileiro de Psiquiatria, Vol. 59, p. 238-246.

Santos, L. S. dos, Ribeiro, Í. J. S., Boery, E. N., \& Boery, R. N. de O. (2017). Qualidade De Vida E Transtornos Mentais Comuns Em Estudantes De Medicina. Cogitare Enfermagem, 22(4), 4-10.

Scheffer, M., Cassenote, A., Guilloux, A. G. A., Miotto, B. A., \& Mainardi, G. M. (2018). Demografia Médica no Brasil 2018.

Serra, R. D., Dinato, S. L. M. e, \& Caseiro, M. M. (2015). Prevalência de sintomas depressivos e ansiosos em alunos de medicina na cidade de Santos. Jornal Brasileiro de Psiquiatria, 64(3), 213-220.

Souza, Marcela Tavares de, Silva, Michelly Dias da, \& Carvalho, Rachel de. (2010). Revisão integrativa: o que é e como fazer. Einstein (São Paulo), 8(1), 102106.

Tabalipa, F. de O., Souza, M. F. de, Pfützenreuter, G., Lima, V. C., Traebert, E., \& Traebert, J. (2015). Prevalence of Anxiety and Depression among Medical Students. Revista Brasileira de Educação Médica, 39(3), 388-394.

Taborda, A. L. de C. G. (2015). Aspectos da resistência do aluno de medicina na busca por auxílio psicológico. Universidade de São Paulo.

Vasconcelos, T. C. de, Dias, B. R. T., Andrade, L. R., Melo, G. F., Barbosa, L., \& Souza, E. (2015). Prevalência de Sintomas de Ansiedade e Depressão em Estudantes de Medicina. Revista Brasileira de Educação Médica, 39(1), 135-142.

Veras, R. M., Fernandez, C. C., Feitosa, C. C. M., \& Fernandes, S. (2020). Perfil Socioeconômico e Expectativa de Carreira dos Estudantes de Medicina da Universidade Federal da Bahia. Revista Brasileira de Educação Médica, 44(2). 